\title{
Practical Application Possibilities for 3D Models Using Low-resolution Thermal Images
}

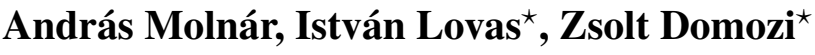 \\ John von Neumann Faculty of Informatics, \\ Óbuda University, H-1034 Budapest, Bécsi út 96/B, Hungary \\ E-mail: molnar.andras@uni-obuda.hu \\ ${ }^{\star}$ Doctoral School of Applied Informatics and Applied Mathematics, \\ Óbuda University, H-1034 Budapest, Bécsi út 96/B, Hungary \\ E-mail: lovas.istvan@uni-obuda.hu,domozi.zsolt@phd.uni-obuda.hu
}

\begin{abstract}
Everyday used cheap thermal cameras can only take low-resolution images. Low-resolution images can be used as the input data of photogrammetry procedures with difficulty or cannot be used at all, as little information is stored of the actual object. Based on the little amount of information, conventional procedures are not capable of identifying a correlation between individual images. Intensity also differs between individual pixels compared to conventional RGB images, thus gradient-based solutions fail to be successful. A method has been developed, which can be used to create thermal image orthophotos from thermal images combined with RGB images. The procedure can result in several output images, from which the most important is the false color thermal image, which is within the visible light spectrum, as on it the original object and the amount of thermal radiation are both visible. Another advantage of the procedure is that not only the information of the visible light spectrum can be visualized but also the data which is invisible to the naked eye.
\end{abstract}

Keywords: thermal photogrammetry; picture fusion; data visualization; $3 D$ objects; low-resolution

\section{Introduction}

Since high resolution thermal cameras are very costly devices, we examined the scenario how photogrammetric procedures can be performed using low resolution thermal camera images. In the course of the experiments several problems and errors had been found, but these were resolved. A procedure was created which resulted in that real thermal image orthophotos and 3D models can be created routinely. The dynamic range of thermal images is significantly lower compared to images taken within visible range. In Figure 1 (a), a stream and its environment can be seen. The photo was taken within a (thermal) range of 7.5-13.5 $\mu \mathrm{m}$. It is clearly visible that the dynamic range of the image is very small. The intensity distribution function seen in 
Figure 1 (a) testifies to a small dynamic range. In Figure 1 (b), we can see the RGB image of the same area at the same time and in the same position. Based on intensity distribution functions it is clearly visible that the dynamic range of individual color channels is much wider compared to the IR image. For the human eye it has more contrast, thus we gain a more satisfactory image.

(a)
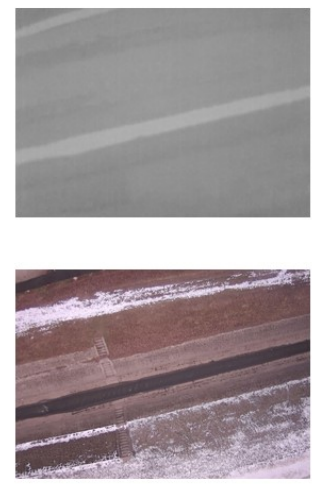
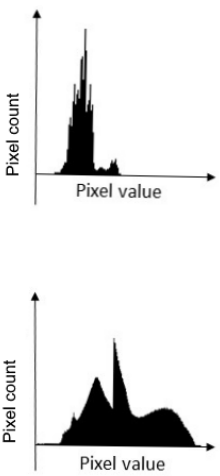

Figure 1

Grey-scale thermo photo with histogram

Low dynamic range images (Figure 1 (a)) not only play a role in the subjective assessment of the image but considering its information content it is of lesser value compared to color images (Figure 1 (b)). In images containing less information it is more difficult to find clearly distinct pixels, thus the efficiency of determining point pairs between the images significantly declines.

The gray image (Figure 1 (a) ) shows the thermal image of a given area with a bit depth 14 (resolution of 1 pixel intensity). In principle, this would mean that the detail of the mapped area (the distinctiveness of the individual pixels) is sufficiently high, i.e. we get a good contrast image. Next to the photo is a histogram of the image that shows how much of the available 14-bit resolution has been used by the camera. Ideally, the entire 14-bit representation range would be covered, but the histogram shows that only less than half of the available range contains data, i.e., a real pixel intensity value. In other words, the image is strongly "underexposed." In the subjective formulation, we feel the same as light and obscure (weak contrast).

In the color image (Figure 1 (b)) shows the RGB image of the area shown in Figure 1 (a). The two recordings were made from the same position at the same time. The grayscale conversion histogram of a color image is a good illustration of the wider range of distribution of each pixel value. Using the above terminology, this means a more detailed, contrasting picture.

Beyond subjective judgment, it can be seen that when searching for point pairs between images, algorithms can use more information for color images or 8-bit grayscale images formed from them than for a higher bit depth but still information-deficient thermal image. 
Processability fundamentally does not depend on the subjective assessment of the images, but on its information content. The pixels of the RGB images applied during the experiments were of $3 \times 8$ bit resolution. In case of thermal images this resolution was $1 \times 14$ bit. As the resolution of the data describing a sole pixel of the RGB image used is substantially higher (24 bit), than the resolution of a sole pixel of the thermal image (14 bit), it makes sense why photogrammetric processing based solely on thermal images was less efficient.

The images seen in Figure 1 and Figure 2 were taken at the same time and in the same position. The grayscale conversion of the image of Figure 1, taken using an RGB camera, can be seen. The images represent a bridge spanning across a stream. In Figure 2, we can see an image taken using an IR camera which represents the object identical to Figure 2. The figures represent two images which were photographed in different positions. [1]

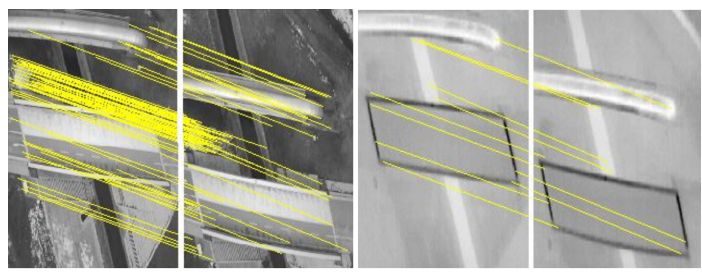

Figure 2

Point pair detection applied on grayscale image pairs using the SIFT algorithm. [2] [3] (left: grayscale from RGB, right: grayscale from IR image)

Figure 2 represents the point pairs detected by the SIFT [4] algorithm. In both cases the parameters of the algorithm were identical. It is apparent that in the grayscale image converted from the RGB image the algorithm found many point pairs, whereas in IR images it found substantially fewer. During the photogrammetric process, these point pairs will supply spatial points. In case of a sufficiently numerous number of point pairs the spatial point cloud will be dense, thus the orthophoto created from it will be rich in detail. In case of few point pairs the point cloud will be very sparse. This, practically, is not sufficient for further processing.

In case of input images containing less information, the photogrammetric process either does not provide a result or provides one with many errors (Figure 3). Photogrammetric processing was done using Agisoft Photoscan. It is visible from the result that during the creation of the point cloud the software made three individual objects which are in fact three sections of a singular object.

Based on the facts introduced above, the photogrammetric procedure not only demands high resolution overlapping images, but it also demands the adequate dynamic of the images. If the pixels possess the necessary amount of information (rich in detail, high contrast, high dynamic range images), and resulting from their resolution many point pairs can be localized in the overlapping areas, successful photogrammetric processing can be expected. With respect to this expectation, we planned the structure of the connection between the color image and the thermal image taken at the same time and in the same location. Based on the aboves this 

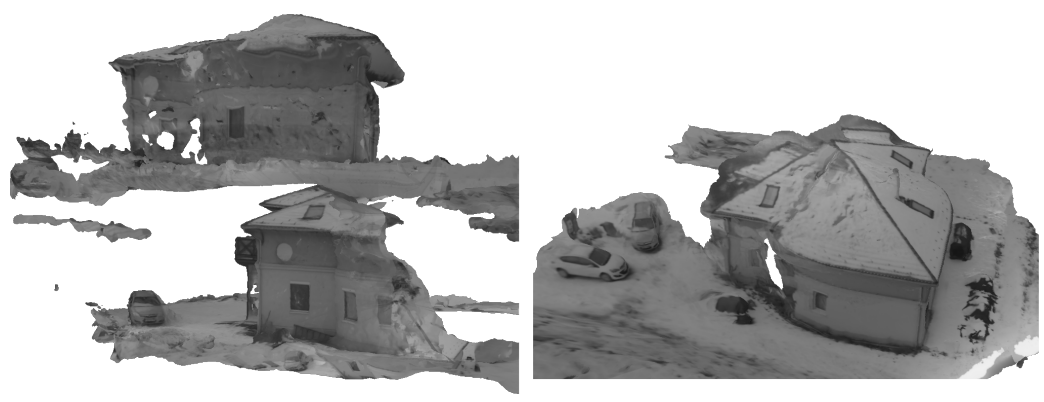

Figure 3

The result of faulty photogrammetric processing

method can be used efficiently on any object which is differ from buildings [5] but it has heat emission like car-parts etc.

The method discussed in this paper is a type of structure from motion and multi-view stereo (SfM-MVS) technique. Essentially, the object under examination is either surrounded by several cameras or they go around it with a single device. The device can be fitted with several tightly or loosely fitting image capture devices to meet special needs. With tight-fitting, the cameras record information received from one other. With loose-fitting, they work independently, and subsequently, during the processing, it is possible to combine and further examine the individual images. The integration of an ordinary camera and a low-resolution thermal imaging camera into one device makes it possible to take high-resolution thermal images of objects using the SfM technique. Our device also produces an RGB and IR image of the area with one shot. These images are not identical, calibration is required.

\section{Materials and Methods}

With conventional photography, distortions due to the structure of the lens are usually not considered. However, with 3D reconstruction, the determination of the parameters of the cameras is essential. The lack of parameters would result in inaccuracy during processing. Two parameters are distinguished; intrinsic and extrinsic parameters. Intrinsic parameters describe the physical characteristics of the camera. These values are independent of the spatial position, always taking a fixed value. It is therefore sufficient to calculate them once during calibration. It is usually given in matrix form:

$$
K=\left[\begin{array}{ccc}
f_{x} & \alpha & c_{x} \\
0 & f_{y} & c_{y} \\
0 & 0 & 1
\end{array}\right]
$$

where $f_{x}, f_{y}$ are focal lengths and $\alpha$ describes the angle between $\mathrm{x}$ and $\mathrm{y}$ axes. 
Another problem can be the radial and tangential distortion caused by the glass lenses in the camera lens. It depends on these parameters, for example, whether the edges of an object, in reality consisting of straight lines, become curved (radial distortion). Extrinsic parameters describe the relationship between the camera system and the world coordinate system, by a rotation matrix $\mathrm{R}$ and a translation vector $\mathrm{T}$.

With an RGB camera, the calibration procedure can be performed using a printed chessboard [6]. The parameters described above can be determined by detecting the corner points of the squares with regular, known dimensions on the chessboard, however, in the case of a thermal imaging camera, this method cannot be used with a paper chessboard. The simple reason for this is that the thermal imaging camera cannot distinguish black and white colors.

A special chessboard was made to calibrate the thermal imaging camera. The black squares of the chessboard are made up of aluminium leaves with good thermal conductivity, which were glued to a white surface with poor thermal conductivity. Before taking the calibration images, the chessboard was heated using a heat source for a few minutes. The result can be seen in the Figure 4 .
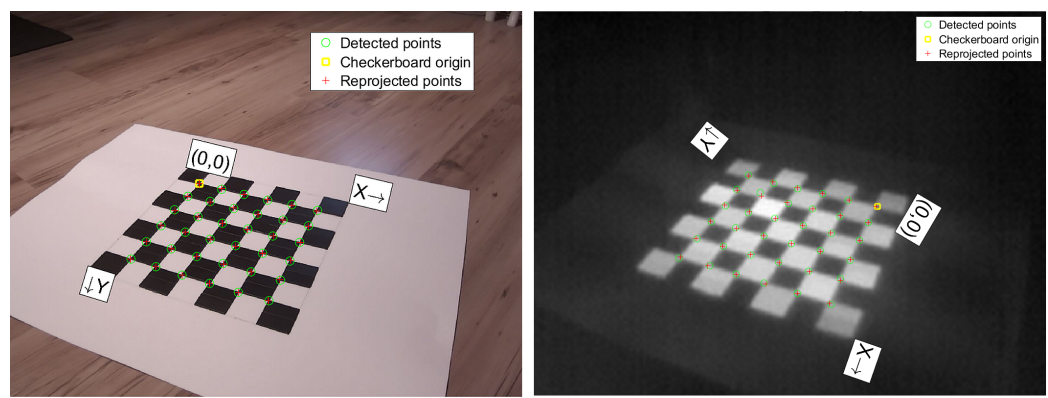

Figure 4

The calibration itself was performed using the Matlab Single Camera Calibrator App

During calibration, the goal is to determine the lens parameters of two different cameras. The image on the left shows the calibration of a color camera, while the image on the right shows the calibration of a thermal camera.

The basis of the procedure created is to take advantage of the connection between the RGB and the IR image. The photogrammetric procedure is performed on the RGB image but the transformations calculated during the procedure will also be performed on the IR image. This is only possible if the two images overlap, in other words, they contain the same area [7]. In this phase, where IR and RGB images reperesenting the same areas, images can be considered as two different data source [8].

As the overlap of RGB and IR images is not ensured, the first phase of our procedure is to create this [9], [10]. Considering the fact that the IR image contains less area than the RGB image, in other words, the content of the IR image is the subset of the RGB image, overlap transformations are performed based on the parameters of the 
IR image. In this procedure, the relative fixed position of the two cameras is taken advantage of. This means that we perform magnification on the IR image based not on image content but on values determined during previous measurements, then, similarly we perform predetermined cropping on the RGB image. As the result of the procedures, RGB-IR image pairs are created which are identical in pixel size and content too. Further conversion is performed on the IR image. The original IR image is a 14 bit one, which with conversion is converted to an 8 bit one. This conversion results in data loss. If the aim is to display relative temperature conditions on the orthophoto or on the 3D model, then this data loss does not cause a substantial error.

Of course, radiometric data vanish as well which means that temperature measurement will not be possible on the processed end result. If data loss is to be avoided, it is possible to extend the 14 bit thermal image to a 24 bit one ( $2 x 8$ bit): the thermal image is $2 \times 7$ bit, which is abrased on $2 \times 8$ bit after the conversation. The $2 \times 8$ bit thermal image information in this case is stored on the green $(G)$ and the blue (B) color channels of the merged image.

With the second step, the RGB image is converted into a grayscale, but color image. This means that all three color channels of the color image contain identical pixel information. The data of the blue (B) color channel of the grey image created this way is replaced with the data of the IR image. As a result, a merged image is created whose one color channel (R) contains the grey image, the other color channel (B) contains the thermal image. The green color channel $(\mathrm{G})$ is not used. [11]

The specially created merged image is suitable for photogrammetric processing. During our experiments, the Agisoft Photoscan software was used. The 3D models or the orthophotos created were subjected to post processing.

During post processing, using the software created, in case of the 3D model we converted the texture images and in case of the orthophoto, the orthophoto itself. Basically, the full color of the image was replaced based on the blue color channel (as this contains the temperature information of the given pixel),. Thus, an artificially colored thermal image is created where the color of the pixel is proportionate to the radation temperature of the original surface. The recoloring program provides an opportunity for the modification of the histogram of the thermal image for the sake of providing a better visual experience. By modifying the histogram a better contrast can be ensured, also small-scale temperature differences can be made visible for the human eye. It is true however, that this phase of post processing does not provide additional information, the visual experience of the result significantly improves.

During the transformations of visible light (RGB) and thermal camera images, rotation was not used. The reason for this is that we assume the exposition of the two cameras occurring at an identical time as well as their relative fixed position. In case of a slowly moving camera system this condition is usually fulfilled. At quick moves (mainly when rotating the camera) however the sync error of the thermal camera and the visible light range camera results in serious deviations regarding the content of the fixed image pairs. Figure 5 represents the image pair "lagging" in time and its merged result. The bottom left image represents the thermal image 


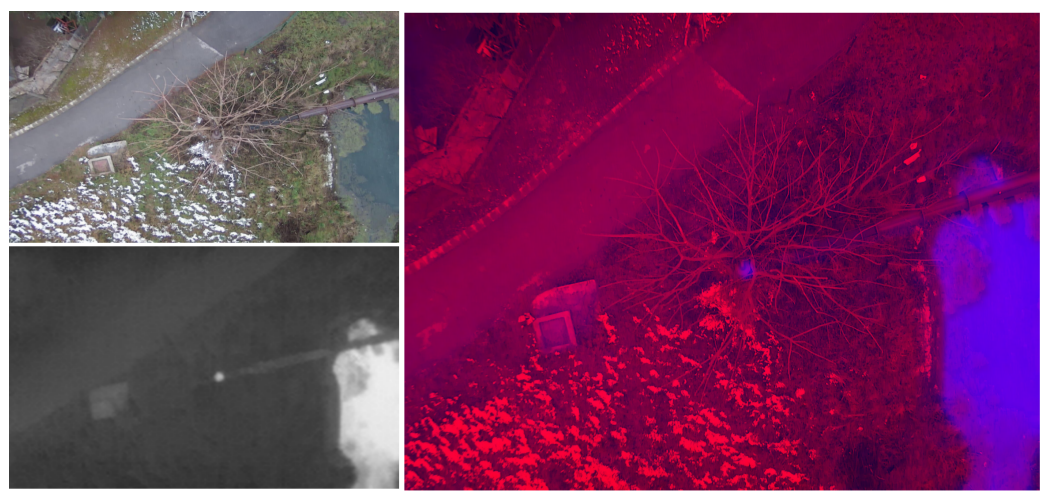

Figure 5

Exposition asynchronicity error

displayed in grayscale, the top left image illustrates the color image of the same area. The large image of Figure 5 is the merged image of the above illustrated image pair. It is visible in the original image pair too that image contents do not match. The result can be observed in the merge image too. As the blue channel of the merged image contains the information of the thermal image the "ghost image" of the source and the spring appears. The image was taken just at the time of the camera's rotation; in this way not only translation but also rotation deviation can be observed too. Such images are to be excluded from post processing. Although it is obvious that mismatched images are not suitable for further processing when shooting at high speed moving, there may be a difference in rotation between the two images.

For the sake of improving visual experience the already prepared temperature colored models (3D model or orthophoto) were improved using edge enhancements. The edges were displayed in the models with white color. This is clearly distinct from the colors containing temperature information and with defined contours it provides a more easily interpretable visual experience. The detection and the enhancement of the edges were done using gradient-based Mamdani fuzzy logic. [12]

As a first step, as it is conventional for edge detecting algorithms, the gradient vector (2) of the grayscale image was determined:

$$
\nabla f=\left[\frac{\partial f}{\partial x} \frac{\partial f}{\partial y}\right]^{T}
$$

In case of digital images, partial derivatives can be approximated with finite difference, with the following general differential equation:

$$
\frac{\partial f}{\partial x}=\lim _{\varepsilon \rightarrow 0}\left(\frac{f(x+\varepsilon, y)}{\varepsilon}-\frac{f(x, y)}{\varepsilon}\right) \approx \frac{f\left(x_{n+1}, y\right)-f\left(x_{n}, y\right)}{\triangle x}
$$


The derivatives can be defined using a convolution mask in $X$ and $Y$ directions. There are a number of possibilities for a convolution mask.

The establishment of a rule base took place in accordance with the following two GMP (Generalized Modus Ponens) conclusions, which contain two antecedents and one consequence:

- "If $I x$ is zero and $I y$ is zero then Iout is white";

- "If $I x$ is not zero or $I y$ is not zero then Iout is black";

where $I x$ is the value of the gradient belonging to axis $X$, while $I y$ is the value of the gradient belonging to axis $Y$.

Membership functions are continuous, trapezoid-shaped functions as seen on Figure 6. For the fuzzy set defuzzification of the conclusion gained as a result, a method of center of gravity location was used.
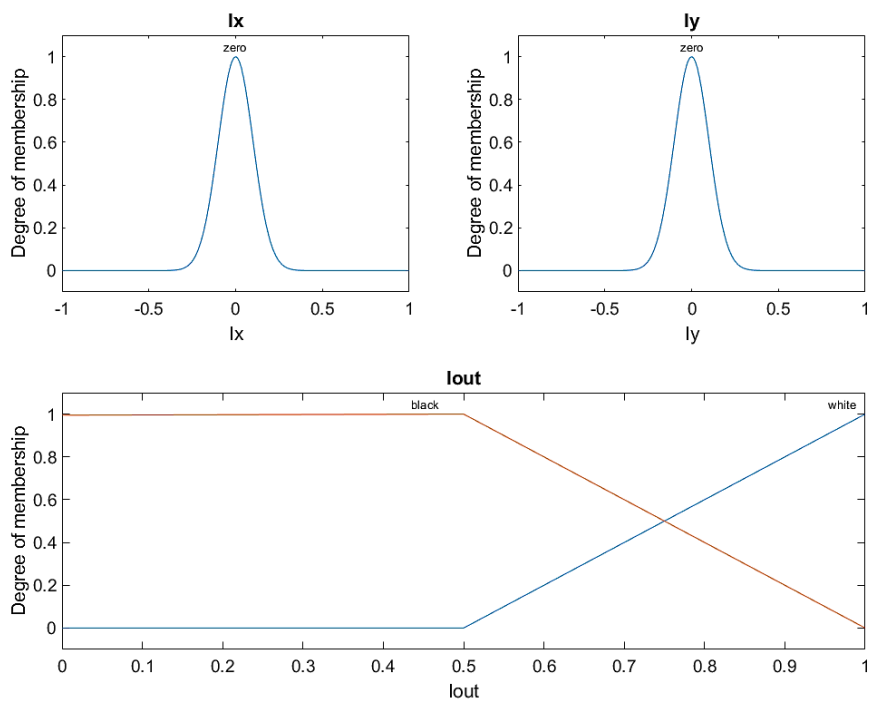

Figure 6

The membership functions of the inputs and the outputs.

The result of merging the image gained after edge enhancement and the palletized thermal image can be seen in Figure 7.

Many different resolution enhancement methods are known, such as RCNN, video stream analysis, or high-resolution image built from time-series images. The problem is that each requires the creation of multiple images, which in many cases is not possible or makes it unnecessarily complicated to produce a high-resolution image.

The presented solution is able to create a high-resolution thermo image from a single image. The procedures presented by others are not capable of this case. 


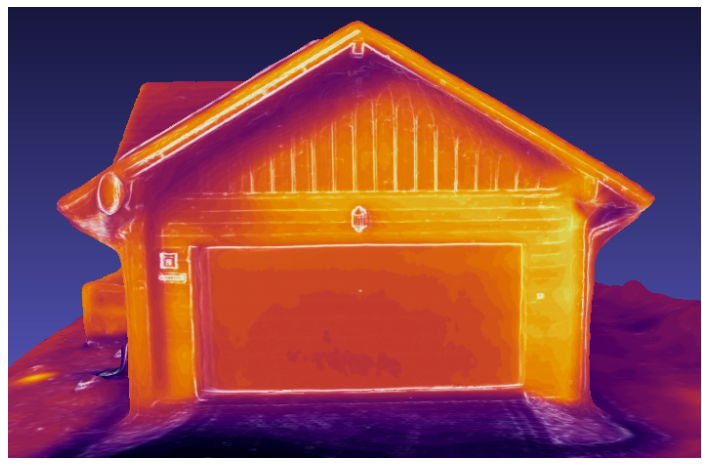

Figure 7

Contoured thermal image produced using fuzzy-based edge detection.

\section{Results}

By utilizing the connection between the color and thermal image taken of the object it became possible to connect the photos in good quality using the photogrammetric method. During the procedure, we created cuts with content identical to the thermal images, which were converted to grayscale images in the next phase. The respective thermal image was connected to the grey scale image using one of the color channels. Thus, the photogrammetric method was performed on specially-made, merged images. At the end of the process, texturing was conducted on the basis of thermal images. [13] In Figure 8, the 3D model of an apartment block can be seen, which was produced using photogrammetry. Grayscale images were used for texturing. 1920x1080 pixel resolution images serve as the basis of the procedure. Texturing was done based on the data of the 3D model, supplied by a thermal camera, which was produced using the procedure described above. As a result, a high quality $3 \mathrm{D}$ thermal model was produced. The resolution of the images of the thermal camera is altogether $160 \times 120$ pixel. In these images it is not possible to detect a sufficient number of high quality feature points, thus they cannot be connected using the photogrammetric method.
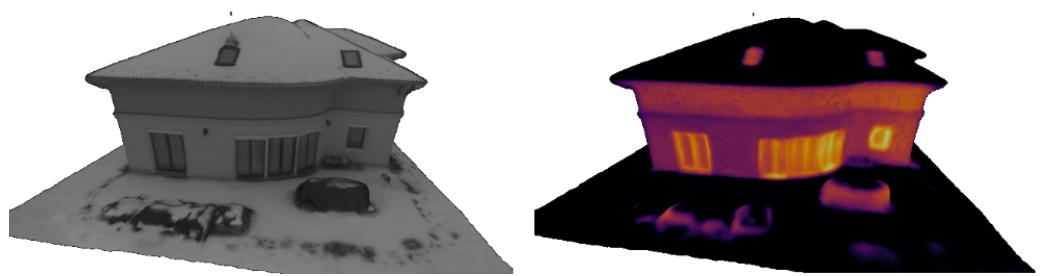

Figure 8

Grayscale and thermal 3D reconstruction of a residential home.

At the photo shoot of the building, the temperature of the environment was $-5{ }^{\circ} \mathrm{C}$. As during the time of the photo shoot the weather was sunny in the few hours before that the camera detected not only the radiation resulting from the temperature loss 
of the house, but also the reflection resulting from the sun's radiation, as well as the back-radiation of its absorbed energy in the form of thermal radiation on the detected thermal image [14].

This is clearly visible on the black objects in front of the building (Figure 8 ). In the thermal image (Figure 8 ), these are warm, though these are not heated objects, only garden furniture covered with black foil protecting them from the weather.

At the border of Dunaalmás, a natural thermal hot spring can be found. Water of 32 ${ }^{\circ} \mathrm{C}$ comes rushing from the depths of the earth which flows into the Danube not far from the spring. 1200 image pairs (RGB and IR) have been created of the area of the spring. The IR orthophoto seen in Figure 9 with $1.5 \mathrm{~cm} /$ pixel field resolution was made using these images. The lightest area of the image can be clearly seen, where the $32{ }^{\circ} \mathrm{C}$ water of the spring rushes to the surface. From this point, the warm spring water flows to the right in the picture. The track of the warm stream looks a little colder in the orthophoto, but in reality, the water in the photographed area does not cool down. Plants are growing on the surface of the stream water and these "shade" surface thermal radiation. During the time of the photo shoot, air temperature was $1{ }^{\circ} \mathrm{C}$.
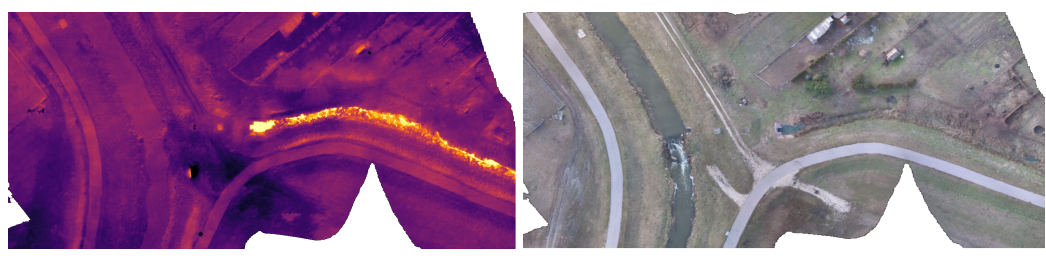

Figure 9

The thermal orthophoto and the color ortophoto of the $32{ }^{\circ} \mathrm{C}$ thermal spring (Dunaalmás)

The color (RGB) orthophoto of the area of the spring can be seen in Figure 9. The spring and the original stream separated from it by a dam can be clearly seen. In the color photo which is natural to the human eye there is not any difference between the two water surfaces, but by comparing the RGB and the IR othophotos, the two water surfaces immediately become clearly distinct.

The colored model of a wooden house can be seen in Figure 10. The model creation procedure was performed in the manner similar to the stone house introduced in Figure 8. It is noticeable in the image that the contours are blurred; regarding the visual experience the image is rather disturbing for the eye. As the observed wooden house was not heated, its thermal image results from the thermal radiation arising from the absorption of the external radiation, as well as from the reflection of the immediate thermal radiation. Hence, the walls are warmer than the windows of the house.

In Figure 10, the 3D model of the wooden house was supplied with contours during post processing. The edges highlighted with white color carry little additional information. In this case, the solar panels engineered on the house become visible during the application of contours. 


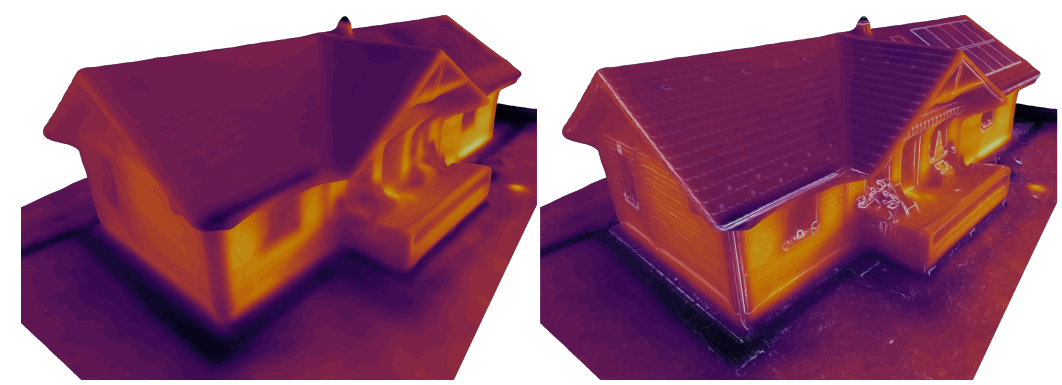

Figure 10

Thermal 3D reconstruction and contoured thermal 3D reconstruction of a wooden house

The model supplied with contours however looks more contrasted; it offers a more pleasant view to the eye. The application of edge enhancement therefore provides a more easily interpretable result in 3D thermal images.

We used Fuzzy edge enhancement, because using Matlab to enhance the visual experience on our data, this was the best procedure.

\section{Discussion}

A 3D thermal image is capable of the thorough examination or inspection of vehicles. Based on the thermal image, the thermal raditation of a passenger car with a closed passenger compartment can be examined. The photos help in the optimization of the planning of the heating system, particularly in the planning of cooling/heating installation. In case of providing sufficient external conditions, satisfactory or just the insufficient heating of the given glass surfaces can be examined (windscreen, side windows). The operation of further heating devices can be examined similarly, by using a thermal image, like for example the heating of side mirrors ( Figure 11) or that of the windscreen.

In Figure 11, the cold air inlet of the engine becomes obvious. Based on the image, it can be seen that the cold air inlet is independent in its total cross-section, in other words an obstruction negatively influencing the cooling of the engine is not visible.

\section{Conclusions}

On the basis of the connection between the thermal and RGB images created of the object, joining images was made possible using photogrammetry. The procedure has created cuts from RGB images equaling the size of the thermal images, and then these images have been converted to grayscale. The procedure used one of the channels of the grayscale image to visualize the thermal image, in this way the details of individual areas of the object and its thermal information became visible. In such images, parts suitable for identification can be found now, based on which the joining has taken place, even by using the photogrammetric method. 


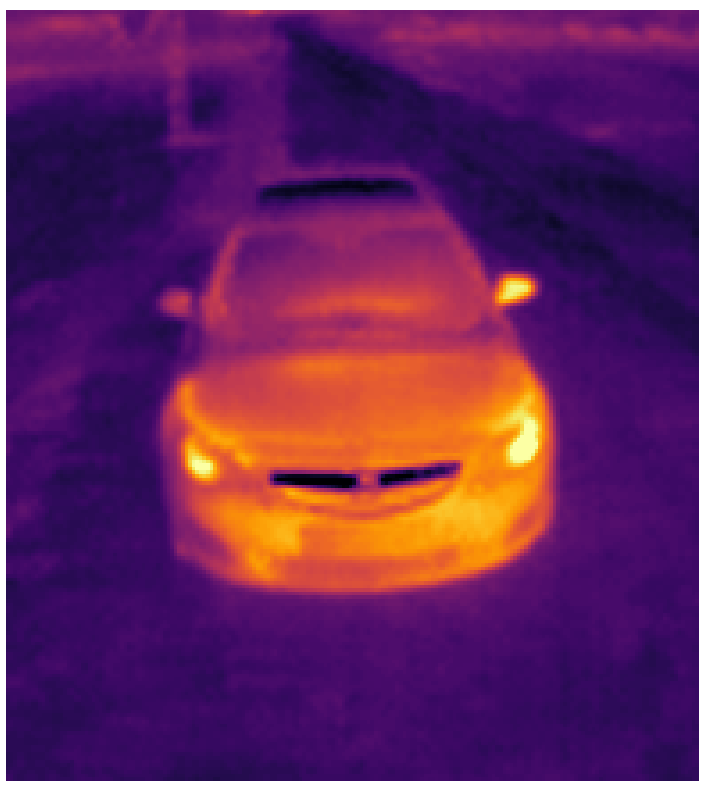

Figure 11

The thermal image of the passenger car in which the heated side mirror on the driver's side can be seen

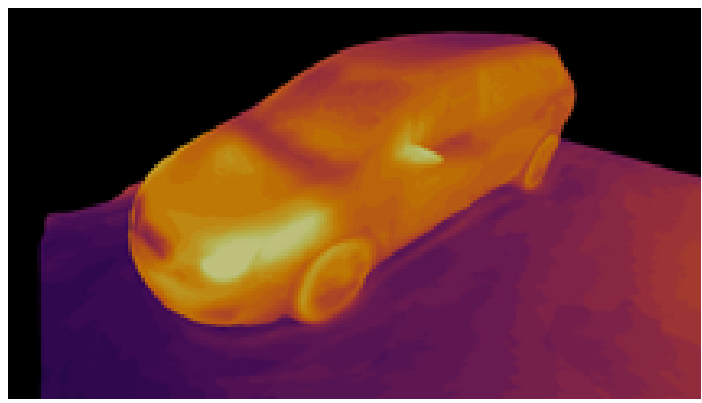

Figure 12

The 3D thermal image of the passenger car

The efficiency of the procedure has been examined on the 3D model of a building and a personal car too. As the basis for testing efficiency, 1920x1080px resolution images were used. Using the above-detailed procedure, texturing took place with the produced images, thus a high-resolution 3D model was created, whose thermal information was gained from low-resolution images, as the resolution of the thermal camera is $160 \mathrm{x} 120 \mathrm{px}$. All elements of the procedure developed have been accomplished using the program Matlab. The Fuzzy edge enhancement implemented also in program Matlab. 


\section{References}

[1] G Kertész, S Szénási, Z Vámossy, "Multi-directional image projections with fixed resolution for object matching“, Acta Polytechnica Hungarica 15 (2), 211-229, 2018.

[2] D. G. Lowe, "Distinctive image features from scale-invariant keypoints", International Journal of Computer Vision, 60, 2 pp. 91-110. 2004.

[3] D. G. Lowe, https://www.cs.ubc.ca/ lowe/keypoints/, 2018.

[4] D. G. Lowe, "Object recognition from local scale-invariant features", Proceedings of the Seventh IEEE International Conference on Computer Vision, 2/8, p. 1150-1157, 1999.

[5] A. Molnar, I. Lovas, Z. Domozi, "Photogrammetry on low resolution thermal pictures", in P. Iványi, B.H.V. Topping, (Editors), "Proceedings of the Sixth International Conference on Parallel, Distributed, GPU and Cloud Computing for Engineering”, Civil-Comp Press, Stirlingshire, UK, Paper 30, 2019. doi:10.4203/ccp. 112.30

[6] Zhang, Zhengyou. "A Flexible New Technique for Camera Calibration." IEEE Trans. Pattern Anal. Mach. Intell. 22 (2000): 1330-1334.

[7] S. Vidas, P. Moghadam, M. Bosse, "3D thermal mapping of building interiors using an RGB-D and thermal camera", 2013 IEEE International Conference on Robotics and Automation, 2013.

[8] Balla, D., Zichar, M., Kozics, A., Mester, T., Mikita, T., Incze, J., Novák, T. J. (2019). A GIS Tool to Express Soil Naturalness Grades and Geovisualization of Results on Tokaj Nagy-Hill. Acta Polytechnica Hungarica, 16(6).

[9] X. ZHAO, J. HE, Y. LUO, N. HUANG and Y. NI, ”Analysis of the Thermal Environment in Pedestrian Space Using 3D Thermography Generated With Unmanned Aerial Vehicles and Infrared Cameras," IGARSS 2019 - 2019 IEEE International Geoscience and Remote Sensing Symposium, Yokohama, Japan, 2019, pp. 4328-4331, doi: 10.1109/IGARSS.2019.8898994.

[10] H. Jung, J. Lyou, "Matching of thermal and color images with application to power distribution line fault detection", Control Automation and Systems (ICCAS) 2015 15th International Conference on, pp. 1389-1392, 2015.

[11] Dorit Borrmann, Florian Leutert, Klaus Schilling, Andreas Nüchter, "Spatial projection of thermal data for visual inspection“, Control Automation Robotics and Vision (ICARCV) 2016 14th International Conference on, pp. $1-6,2016$.

[12] M. Takacs, "Mamdani-type Implication Inference with Degree of Coincidence“, (2003) 1st Slovakian - Hungarian Symposium on Applied Machine Intelligence (SAMI 2003), Herlany, Slovakia, February 12-14, ISBN. 9647154140, pp.67-73., 2003. 
[13] M. Scaioni, E. Rosina, L. Barazzetti, M. Previtali, V. Redaelli, "High-resolution texturing of building facades with thermal images", Proc. SPIE 8354, Thermosense: Thermal Infrared Applications XXXIV, 83540I (18 May 2012); doi: 10.1117/12.920613;

[14] M.C. Harvey, J.V. Rowland, K.M. Luketina, ”Drone with thermal infrared camera provides high resolution georeferenced imagery of the Waikite geothermal area, New Zealand", Journal of Volcanology and Geothermal Research, Volume 325, Pages 61-69, 2016. 